\title{
Missing baryons in shells around galaxy clusters
}

\author{
D. A. Prokhorov ${ }^{1,2}$ \\ 1 Institut d'Astrophysique de Paris, CNRS, UMR 7095, Université Pierre et Marie Curie, 98bis Bd Arago, 75014 Paris, France \\ e-mail: prokhoro@iap.fr \\ 2 Moscow Institute of Physics and Technology, Institutskii lane, 141700 Moscow Region, Dolgoprudnii, Russia
}

Accepted 30 June 2008 / Accepted 23 October 2008

\section{ABSTRACT}

\begin{abstract}
Aims. The cluster baryon fraction is estimated from the CMB-scattering leptonic component of the intracluster medium (ICM); however, the observed cluster baryon fraction is less than the cosmic one. Understanding the origin of this discrepancy is necessary for correctly describing the structure of the ICM.

Methods. We estimate the baryonic mass in the outskirts of galaxy clusters which is difficult to observe because of low electron temperature and density in these regions.

Results. The time scale for the electrons and protons to reach equipartition in the outskirts is longer than the cluster age. Since thermal equilibrium is not achieved, a significant fraction of the ICM baryons may be hidden in shells around galaxy clusters. We derive the necessary condition on the cluster mass for the concealment of missing baryons in an outer baryon shell and show that this condition is fulfilled because cluster masses are comparable to the estimated characteristic mass $M=e^{4} /\left(m_{\mathrm{p}}^{3} G^{2}\right)=1.3 \times 10^{15}$ solar masses. The existence of extreme-ultraviolet emission haloes around galaxy clusters is predicted.
\end{abstract}

Key words. galaxies: clusters: general - galaxies: intergalactic medium - cosmology: cosmological parameters - ultraviolet: general

\section{Introduction}

Clusters of galaxies are the largest virialized structures in the Universe. They typically contain hundreds of galaxies, spread over a region whose size is roughly $3 \mathrm{Mpc}$. Their total masses are about $10^{15} M_{\odot}$. The space between galaxies in clusters is filled with very hot $\sim 10^{8} \mathrm{~K}$, low-density $\sim 10^{-3} \mathrm{~cm}^{-3}$ gas (for a review, e.g. Sarazin 1986).

It is widely believed that clusters should be representative of baryonic and nonbaryonic matter compositions, and thus that their baryon fractions can be used to determine the average density of the Universe in conjunction with Big Bang nucleosynthesis (e.g. White et al. 1993).

Recent good quality observations of massive clusters with Chandra and XMM-Newton put strong observational constraints on the gas mass fraction $f_{\mathrm{b}}$ in massive clusters at large radius (e.g. Allen et al. 2004; Zhang et al. 2006). In many cases the new data allow this fraction to be constrained out to the radius $r_{500}$ at which the density contrast is 500 . The observable baryon fraction is lower than the best fit to the WMAP 5-year result of $f_{\mathrm{b}}=0.17 \pm 0.01$ (Dunkley et al. 2008).

$\mathrm{X}$-ray observations are not the only way to constrain the gas mass fractions of clusters. The Sunyaev-Zeldovich (SZ) effect is also being used for this purpose. LaRoque et al. (2006) estimated $f_{\mathrm{b}}$, in the radius at which the density contrast is 2500 , by fitting both BIMA/OVRO SZ effect data and Chandra X-ray data simultaneously and fitting the SZ effect data alone. These results are in excellent agreement with the X-ray derived results.

Measurements of the SZ effect in the CMB maps of WMAP data (Afshordi et al. 2007), through study of a sample of 193 massive galaxy clusters with observed X-ray temperature greater than $3 \mathrm{keV}$, indicate that a significant fraction, $35 \% \pm 8 \%$, of baryonic mass is missing from the hot ICM, and thus must cool to form galaxies, intracluster stars, or an unknown cold phase of the ICM. There does not seem to be enough mass in the form of stars or cold gas in the cluster galaxies or intracluster space, signaling the need for a still unknown baryonic component, or otherwise new astrophysical processes in the ICM (Afshordi et al. 2007).

Much larger discrepancies with X-ray observations have been reported in Lieu et al. (2006), who claim that the WMAP SZ signal for ROSAT X-ray clusters is a factor of 4 weaker than the expectation from X-ray brightness profiles.

However, in cosmological simulations the baryon fraction within $r_{500}$ has converged to $90-95 \%$ of the best fit to the WMAP 5 -year data (Dunkley et al. 2008) in the case of non-radiative simulations (e.g. Frenk et al. 1999; Kay et al. 2004; Crain et al. 2006). Cosmological cluster simulations that include gas cooling and star formation also give an ICM mass fraction very similar to the WMAP 5-year fraction (Kravtsov et al. 2005).

In a hierarchical Universe clusters form through accretion. As fresh material joins the stationary cluster gas, it converts its infall kinetic energy into heat through a shock. A shock front moves outward as ambient gas accretes toward the cluster center (see e.g. Takizawa \& Mineshige 1998). The accretion shock around an X-ray cluster primarily heats the protons, since they carry most of the kinetic energy of the infalling gas. The electrons, on the other hand, remain cold after the shock with their temperature slowly rising as a result of their Coulomb collisions with the hot ions. Thermal equilibrium will only be achieved if the temperature equilibrium time between the protons and the electrons is shorter than the age of the cluster (e.g. Fox \& Loeb 1997).

The Sunyaev-Zeldovich distortion of the CMB spectrum is proportional to the electron temperature. Thus, the low gas fractions inferred from the SZ effect observations in the CMB maps of WMAP data (Lieu et al. 2006; Afshordi et al. 2007) can be interpreted as evidence of a warm phase (with low electron 
temperature $T_{\mathrm{e}} \sim 0.1 \mathrm{keV}$ and high proton temperature) of the ICM in the cluster outskirts, which would not contribute appreciably to the SZ signal, while containing a significant fraction of the ICM baryons.

The importance of cluster outskirt analysis is discussed in Sect. 2, where the baryon fraction profile is calculated in the case of a simple isothermal beta model. The formation of baryonic shells around clusters is considered within the framework of kinetic theory in Sect. 3. A condition on the cluster mass for deviation from thermal equilibrium is derived in Sect. 4. The influence of the deviation from thermal equilibrium on the prediction for the SZ decrement is considered in Sect. 5. A method for detecting missing baryons by means of EUV astronomy is proposed in Sect. 6 and a discussion is presented in Sect. 7.

\section{Tracing the baryon fraction in the outskirts within the framework of the hydrostatic theory}

The ICM emits energy mainly via thermal bremsstrahlung, which is proportional to the density squared. Therefore external cluster regions show less X-ray emission than the central ones, where the density is much higher. This lower emission translates in lower statistics for available X-ray observations. One possible way to overcome the problem of low statistics in the outer regions is to fit clusters with the isothermal beta model (Cavaliere \& Fusco-Femiano 1976). The disadvantage of such a method is clear: the extrapolation depends on the validity of the model in the cluster outskirts. In accordance with the beta model, the electron number density follows the spherical distribution

$n(r)=n_{0}\left(1+\frac{r^{2}}{r_{\mathrm{c}}^{2}}\right)^{-\frac{3 \beta}{2}}$

where $n_{0}$ is the central number density, $r_{\mathrm{c}}$ is the core radius, $\beta$ is the slope parameter.

The hydrostatic equilibrium equation for a spherically symmetric isothermal cluster (e.g. Sarazin 1986) can be written as:

$k T \mathrm{~d} n=-\frac{G M(R) \mu m_{\mathrm{p}} n(R) \mathrm{d} R}{R^{2}}$

where $M(R)=\int_{0}^{R} 4 \pi r^{2} \psi(r) \mathrm{d} r$ is the total mass within a sphere of radius $R, \psi(r)$ is the mass density of all the matter, $\mu$ the mean molecular weight, $m_{\mathrm{p}}$ the proton mass.

The baryonic fraction $f_{\mathrm{b}}(r)=\rho(r) / \psi(r)$ is given by

$f_{\mathrm{b}}(r)=-\frac{4 \pi \mu m_{\mathrm{p}} G}{k T} \frac{\rho(r) r^{2}}{\left(r^{2} \rho^{-1}(r) \cdot \mathrm{d} \rho(r) / \mathrm{d} r\right)^{\prime}}$

where $\rho(r)=m_{\mathrm{p}} n(r)$ is the gas mass density, the prime denotes a derivation of $r$.

Using Eq. (1), one can calculate the ratio $f_{\mathrm{b}}(r) / f_{\mathrm{b}}\left(r_{\mathrm{c}}\right)$ for the isothermal beta model

$F(r)=\frac{f_{\mathrm{b}}(r)}{f_{\mathrm{b}}\left(r_{\mathrm{c}}\right)}=2^{3 \beta / 2}\left(1+\frac{r^{2}}{r_{\mathrm{c}}^{2}}\right)^{2-3 \beta / 2} \times\left(3+\frac{r^{2}}{r_{\mathrm{c}}^{2}}\right)^{-1}$.

The maximum of the function $F(r)$ is at the point $a=r / r_{\mathrm{c}}$ :

$a=\sqrt{\frac{9 \beta-10}{2-3 \beta}}, \quad 2 / 3<\beta<10 / 9$.

The maximum value of $F(r)$ is given by

$F \max =\sqrt{(4-3 \beta)^{4-3 \beta}(3 \beta-2)^{3 \beta-2}}$.

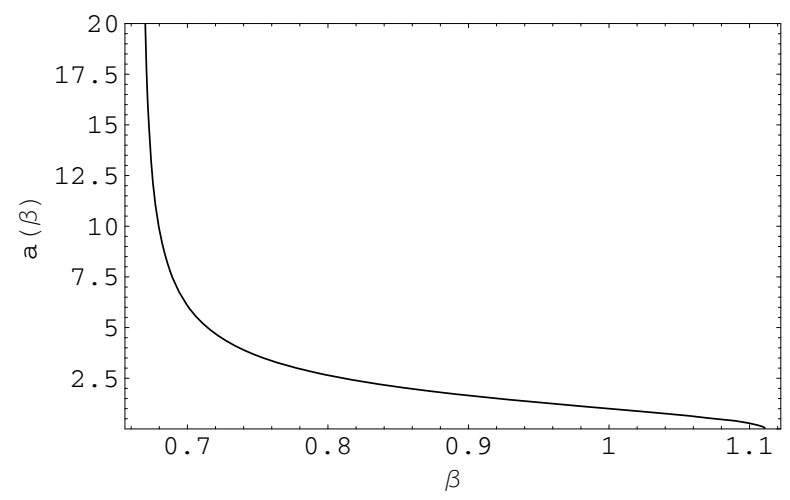

Fig. 1. Position of the maximum of the $f_{\mathrm{b}}(r) / f_{\mathrm{b}}\left(r_{\mathrm{c}}\right)$ ratio as a function of the slope parameter $\beta$.

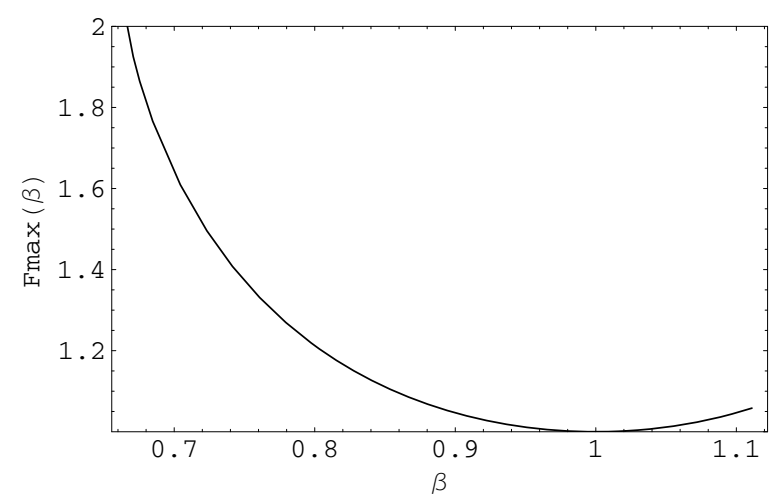

Fig. 2. Maximum value of the $f_{\mathrm{b}}(r) / f_{\mathrm{b}}\left(r_{\mathrm{c}}\right)$ ratio as a function of the slope parameter $\beta$.

The dependence $a(\beta)$ and the maximum value of $F \max (\beta)$ are plotted in Figs. 1 and 2 respectively.

If $\beta<2 / 3$ then the baryon fraction $F(r)$ is an increasing function of the radius. The average value of $\beta$ found by Jones $\&$ Forman (1984) from the X-ray distribution is $\beta=0.65$, which implies that the gas is considerably more extensively distributed than the mass in the cluster and the baryon fraction in the outskirts is higher than in the central region. Hence a study of the cluster outskirts is essential for understanding the baryon component distribution in clusters.

The mass of the gas shell between the accretion shock radius around a galaxy cluster and the virial radius $R_{\text {vir }}$ in the context of the beta model (with $\beta<1$ and $r_{\mathrm{c}} \ll R_{\mathrm{vir}}$ ) is given by integrating Eq. (1):

$M_{\text {shell }}^{\text {gas }} \approx M^{\text {gas }}\left(R_{\text {vir }}\right) \times\left(\alpha^{3-3 \beta}-1\right)$

where $M^{\mathrm{gas}}\left(R_{\mathrm{vir}}\right)$ is the gas mass inside the virial radius sphere, and $\alpha$ is the ratio of the shock radius to the virial radius.

Lieu et al. (2006) claim that the WMAP SZ signal for ROSAT X-ray clusters is much weaker than the expectation from the X-ray brightness profile. In order to compare with WMAP maps, they extrapolate the isothermal beta model fits far beyond the region that is fit by X-ray data. The reported discrepancy may be evidence of a warm phase of the ICM in the cluster outskirts with electron temperature $T_{\mathrm{e}} \sim 0.1 \mathrm{keV}$, which would not contribute appreciably to the SZ signal, and the proton temperature is of the order of the virial one (see Sect. 5). 


\section{Formation of a baryonic shell within the framework of the kinetic theory}

In accordance with the hierarchical clustering scenario, the baryons are accreted along with the dark matter onto a galaxy cluster. An accretion shock at the interface of the inner hydrostatic gas with a cooler external medium is a long-standing prediction from gravitationally driven models (e.g., see the model of Bertschinger 1985).

The accretion shock converts the radial motion energy of the upstream protons into random motion energy, below the shock. Let us assume that the protons below the accretion shock are essentially collisionless and have large orbital eccentricities. Then they are able to accrete into the X-ray cluster on the Coulomb time scale, since this is the time in which they are able to dissipate their excess orbital angular momentum (for a similar view of a quasar model, see Meszaros \& Ostriker 1983). If this time scale is comparable with the cluster age, then a considerable part of the protons can be located between the accretion shock and the inner collisional plasma region. The Coulomb mean-freepath of protons is larger than the virial radius of the cluster at temperature $\gtrsim 2 \mathrm{keV}$ (Loeb 2007), therefore it is reasonable to choose the virial radius as the inner collisional region border.

The Coulomb mean-free-path of a proton in a plasma of temperature $T$ is given by (Spitzer 1956):

$l=\frac{3^{3 / 2} k_{\mathrm{B}}^{2} T^{2}}{4 \pi^{1 / 2} n e^{4} \ln \Lambda}$

where $T$ is the proton temperature, $n$ is the proton number density, $\ln \Lambda$ is the Coulomb logarithm, $e$ is the electron charge, $k_{\mathrm{B}}$ is the Boltzmann constant.

The proton random energy (or "temperature") is of the order of the gravitational temperature.

$T_{\mathrm{gr}}=\frac{G M\left(R_{\mathrm{vir}}\right) m_{\mathrm{p}}}{k_{B} R_{\mathrm{vir}}}$

where $M\left(R_{\mathrm{vir}}\right)$ is the total mass inside the sphere of radius $R_{\mathrm{vir}}$, which equals (e.g. Eke et al. 1998)

$R_{\mathrm{vir}}=\left(\frac{3 M\left(R_{\mathrm{vir}}\right)}{4 \pi \Delta_{\mathrm{c}} \rho_{\mathrm{cr}}}\right)^{1 / 3}$

where $\Delta_{c}$ is the density contrast for the formation of the cluster.

The protons will be collisionless if the Coulomb mean-free path $l$ is higher than the distance $L$, which the proton can cover during the shock propagation time $t=\tau H_{0}^{-1}$ from the virial radius to the present accretion shock radius:

$l>L$

where $H_{0}$ is the Hubble constant.

It is convenient to rewrite the distance $L$ by means of Eqs. (9) and (10):

$L=\tau H_{0}^{-1} \sqrt{\frac{k_{\mathrm{B}} T_{\mathrm{gr}}}{m_{\mathrm{p}}}}=\tau R_{\mathrm{vir}} \sqrt{\frac{\Delta_{\mathrm{c}}}{2}}$.

Using Eqs. (8) and (11), one can show that the collisionless condition is equivalent to the inequality

$n<\frac{3^{3 / 2} k_{\mathrm{B}}^{2} T^{2}}{4 \pi^{1 / 2} L e^{4} \ln \Lambda}$
The mass of the gas shell between the accretion shock and virial radius is

$M_{\text {shell }}^{\text {gas }}=\frac{4 \pi}{3} R_{\text {vir }}^{3}\left(\frac{R_{\mathrm{s}}^{3}}{R_{\mathrm{vir}}^{3}}-1\right) n m_{\mathrm{p}}$

where $R_{\mathrm{S}}$ is the present shock radius.

The overdensity in Bertschinger's collisionless model, given by the dimensionless quantity $M(\lambda) / \lambda^{3}$, equals 178 at $\lambda_{\text {vir }} \approx$ 0.255 (Fox \& Loeb 1997), where the dimensionless parameter $\lambda=r / r_{\mathrm{ta}}$ and $r_{\mathrm{ta}}$ is the turnaround radius. The shock occurs at fixed $\lambda_{\mathrm{s}} \approx 0.347$ (for $\gamma=5 / 3$ ) in accordance with the shocked accretion model of a collisional gas (Bertschinger 1985). Consequently, the ratio of the shock radius to the virial radius $\alpha=R_{\mathrm{S}} / R_{\text {vir }}$ equals 1.36 .

One can rewrite the condition $l>L$ in terms of the shell mass

$M_{\text {shell }}^{\text {gas }}<\frac{4 \pi}{3}\left(\alpha^{3}-1\right) R_{\mathrm{vir}}^{3} m_{\mathrm{p}} \frac{3^{3 / 2} k_{\mathrm{B}}^{2} T^{2}}{4 \pi^{1 / 2} e^{4} L \ln \Lambda}$

replacing $T$ by $T_{\mathrm{gr}}$ from Eq. (9) one can get:

$M_{\text {shell }}^{\text {gas }}<\tau^{-1} \sqrt{\frac{6 \pi}{\Delta_{\mathrm{c}}}}\left(\alpha^{3}-1\right) m_{\mathrm{p}}^{3} \frac{G^{2} M^{2}\left(R_{\mathrm{vir}}\right)}{e^{4} \ln \Lambda}$.

If the missing baryons are situated in the collisionless shell, one can evaluate the value $M_{\text {shell }}^{\text {gas }} / M^{\text {gas }}\left(R_{\text {vir }}\right)=\left(f_{\mathrm{b}}-f\right) / f$, where $f=$ $M^{\text {gas }}\left(R_{\text {vir }}\right) / M\left(R_{\text {vir }}\right)$ and $f_{\mathrm{b}}$ is the best fit to the WMAP 5-year result. Therefore $M_{\text {shell }}^{\text {gas }} / M\left(R_{\text {vir }}\right)=f_{\mathrm{b}}-f$.

Thus a collisionless shell should exist if the total cluster mass satisfies the necessary condition

$M\left(R_{\mathrm{vir}}\right)>\tau \sqrt{\frac{\Delta_{c}}{6 \pi}} \frac{\left(f_{\mathrm{b}}-f\right) \ln \Lambda}{\alpha^{3}-1} \frac{e^{4}}{G^{2} m_{\mathrm{p}}^{3}}$.

The time evolution of the radius $r_{\mathrm{s}}(t)$ of a shock surface follows $r_{\mathrm{s}}(t) \propto t^{8 / 9}$ (Bertschinger 1985). The dimensionless shock propagation time from the virial radius to the present accretion shock radius equals $\tau=1-\alpha^{-9 / 8}$, assuming here that the cluster age is the Hubble time.

If $f_{\mathrm{b}}-f=0.056$ (if $33 \%$ of baryons are missing), $\alpha=1.36$ (the ratio of the shock radius to the virial radius in Bertschinger's model) and $\tau=0.29$ (the shock propagation time), then the total cluster mass must exceed the limit mass value

$M\left(R_{\text {vir }}\right)>1.2 \times 10^{15} M_{\odot}$.

X-ray measurements tend to come from the inner parts of clusters. One way to relate an observable quantity in the inner regions of a cluster to a quantity in the outer regions is to use a simplified model, such as that considered by Eke et al. (1996), in which both the X-ray gas and the total mass have the form of a singular isothermal sphere in the virialized section of the cluster. In this model, the mass within the sphere of the virial radius and the temperature of the X-ray gas are related by

$M\left(R_{\mathrm{vir}}\right)=1.7 \times 10^{15} h_{70}^{-1}\left(\frac{T_{\mathrm{X}}}{10^{8} \mathrm{~K}}\right)^{3 / 2} M_{\odot}$

where $h_{70}$ is the Hubble constant in units of $70 \mathrm{~km} \mathrm{~s}^{-1} \mathrm{Mpc}^{-1}$.

The limit mass value $\left(1.2 \times 10^{15} M_{\odot}\right)$ is comparable with a typical cluster mass, therefore a fraction of baryons can be trapped in the baryonic shell around clusters within the framework of kinetic theory. 


\section{Deviation from thermal equilibrium}

In Sects. 2 and 3 using both hydrostatic and kinetic theories we conclude that the fraction of cluster baryons located in the outskirts may be significant. This raises the possibility that a missing fraction of baryonic mass may be hidden in the cluster outskirts where the electron temperature is much smaller than the proton one, and therefore the contribution of these electrons to the SZ signal is small.

The time scale $t_{\text {eq }}(\mathrm{p}, \mathrm{e})$ for the electrons and protons to reach equipartition $T_{\mathrm{e}}=T_{\mathrm{p}}$ is given by $t_{\mathrm{eq}}(\mathrm{p}, \mathrm{e}) \approx \sqrt{m_{\mathrm{p}} / m_{\mathrm{e}}} \cdot t_{\mathrm{eq}}(\mathrm{p}, \mathrm{p})$ (e.g. Sarazin 1986), where the time scale $t_{\mathrm{eq}}(\mathrm{p}, \mathrm{p})=l / \sqrt{k T_{\mathrm{gr}} / m_{\mathrm{p}}}$ for protons to equilibrate among themselves .

The electrons will be cold between the accretion shock and virial radius if the time scale $t_{\mathrm{eq}}(\mathrm{p}, \mathrm{e})$ is longer than the shock propagation time $t=\tau H_{0}^{-1}$ from the virial radius to the present accretion shock radius, i.e. $t_{\mathrm{eq}}(\mathrm{p}, \mathrm{e})>\tau H_{0}^{-1}$. This condition can be rewritten in the form equivalent to Eq. (11):

$l>\sqrt{\frac{m_{\mathrm{e}}}{m_{\mathrm{p}}}} \cdot L$

Assuming that the missing baryons are situated in the shell between the accretion shock and virial radii and following the same method as in Sect. 3, one can derive a necessary condition for deviation from thermal equilibrium:

$M\left(R_{\mathrm{vir}}\right)>\tau \sqrt{\frac{m_{\mathrm{e}}}{m_{\mathrm{p}}}} \sqrt{\frac{\Delta_{\mathrm{c}}}{6 \pi}} \frac{\left(f_{\mathrm{b}}-f\right) \ln \Lambda}{\alpha^{3}-1} \frac{e^{4}}{G^{2} m_{\mathrm{p}}^{3}}$.

If $f_{\mathrm{b}}-f=0.056$ (if $33 \%$ of baryons are missing), $\alpha=1.36$ (the ratio of the shock radius to the virial radius in Bertschinger's model) and $\tau=0.29$ (the shock propagation time), then the numerical value of this mass is

$M\left(R_{\text {vir }}\right)>2.8 \times 10^{13} M_{\odot}$.

The value of the limit mass $M\left(R_{\text {vir }}\right)=2.8 \times 10^{13} M_{\odot}$ is smaller than cluster masses for a sample that was used by Afshordi et al. (2007) and therefore the missing baryons may be hidden in shells around galaxy clusters. Note that even for a value $\tau=1$ (the cluster age) the limit mass is still smaller than cluster masses for the Afshordi et al. (2007) sample.

This explanation of the origin of the missing baryonic mass in the clusters arises from a coincidence between a typical cluster mass and a characteristic mass $\left(1.3 \times 10^{15} M_{\odot}\right)$, which is given by

$M=\frac{e^{4}}{m_{\mathrm{p}}^{3} G^{2}}$.

The characteristic mass depends only on fundamental physical constants.

\section{Prediction for the SZ decrement}

The observed SZ effect with WMAP is much less than the predicted decrement value given by the isothermal beta model (Lieu et al. 2006). Their study used a single-temperature model for the intracluster medium, under the assumption that equipartition is fully achieved. However, the outskirts of galaxy clusters actually have a two-temperature structure, because the two temperatures, i.e. those of electrons $T_{\mathrm{e}}$ and protons $T_{\mathrm{p}}$, remain different (e.g. Fox \& Loeb 1997). To understand the discrepancy between observed and predicted SZ decrements it is useful to study the influence of the deviation from thermal equilibrium on the prediction for the SZ decrement.

The thermal SZ effect (see Sunyaev \& Zeldovich 1980, for a review) describes the inverse Compton scattering of CMB photons by the hot gas in clusters. The resulting change in the (thermodynamic) CMB temperature observed at frequency $v$ is given by

$\Delta T_{v}=y j(x) T_{0}$

where $T_{0}=2.725 \mathrm{~K}$ is the unperturbed CMB temperature, $y$ is the Comptonization parameter, $x$ is a dimensionless parameter defined as $x=h v / k T_{0}$ and in the nonrelativistic regime $\left(k T_{\mathrm{e}} \ll\right.$ $\left.m c^{2}\right)$

$j(x)=x \frac{e^{x}+1}{e^{x}-1}-4$.

The Comptonization parameter is

$y=\int \mathrm{d} l \frac{k T_{\mathrm{e}}}{m c^{2}} \sigma_{\mathrm{T}} n$

where $T_{\mathrm{e}}$ is the electron temperature, $\sigma_{\mathrm{T}}$ is the Thomson cross section, and the integral is over the distance along the line-ofsight.

The total flux $S_{v}$ of a cluster (e.g. Refregier et al. 2000) observed at frequency $v$ is related to the angular integral of the thermodynamic temperature shift $\Delta T_{v}$ by

$S_{v}=\frac{2 k^{3} T_{0}^{2}}{h^{2} c^{2}} q(x) \int \mathrm{d} \Omega \Delta T_{v}$

where $q(x)=x^{4} /[2 \sinh (x / 2)]^{2} .{ }^{1}$ For the WMAP W channel $\left(v=94 \mathrm{GHz}, x_{94}=1.65\right): q\left(x_{94}\right)=2.18$ and the full width at half maximum (FWHM) equals 10 arcmin.

The postshock protons carry nearly all of the bulk kinetic energy. After passing through a shock the electrons can gain thermal energy via collisions with protons. The evolution of the electron temperature is given by

$\frac{\mathrm{d} T_{\mathrm{e}}}{\mathrm{d} t}=\frac{T_{\mathrm{p}}-T_{\mathrm{e}}}{t_{\mathrm{eq}}(\mathrm{p}, \mathrm{e})}$.

The collision time scale $t_{\mathrm{eq}}(\mathrm{p}, \mathrm{e})$ (Spitzer 1958) equals

$t_{\mathrm{eq}}(\mathrm{p}, \mathrm{e})=\frac{3 m_{\mathrm{e}} m_{\mathrm{p}}}{8 \sqrt{2 \pi} n e^{4} \ln \Lambda}\left(\frac{k_{B} T_{\mathrm{e}}}{m_{\mathrm{e}}}+\frac{k_{B} T_{\mathrm{p}}}{m_{\mathrm{p}}}\right)^{3 / 2}$.

Using the dependence of the shock radius $r_{\mathrm{s}}(t) \propto r^{8 / 9}$ from the work of Bertschinger (1985) and Eqs. (28), (29) and assuming typical values of the central electron temperature $k_{\mathrm{B}} T_{0}=8 \mathrm{keV}$, of the central density $n_{0}=10^{-3} \mathrm{~cm}^{-3}$, and of the slope parame$\operatorname{ter} \beta=2 / 3$, we found the electron temperature profile and calculated both the SZ temperature decrement and the total SZ flux $S_{v}$ within $10^{\prime}$ radius for the non-isothermal beta model. For a cluster with a core radius $0.5^{\prime}$ which corresponds to the mean core radius in the sample of clusters by Bonamente et al. (2006), the SZ decrements are plotted in Fig. 3 for the isothermal and nonisothermal beta models. The total SZ flux of a cluster in the nonisothermal model is smaller by a factor of 2.6 than those computed with a single-temperature model. The SZ decrement for the isothermal beta model which is scaled in such a way that the total SZ flux corresponds to the non-isothermal model is also plotted in Fig. 3.

\footnotetext{
1 Note that this is the correct formula, while there is a misprint in the expression of $q(x)$ in Eq. (19) by Refregier et al. (2000).
} 


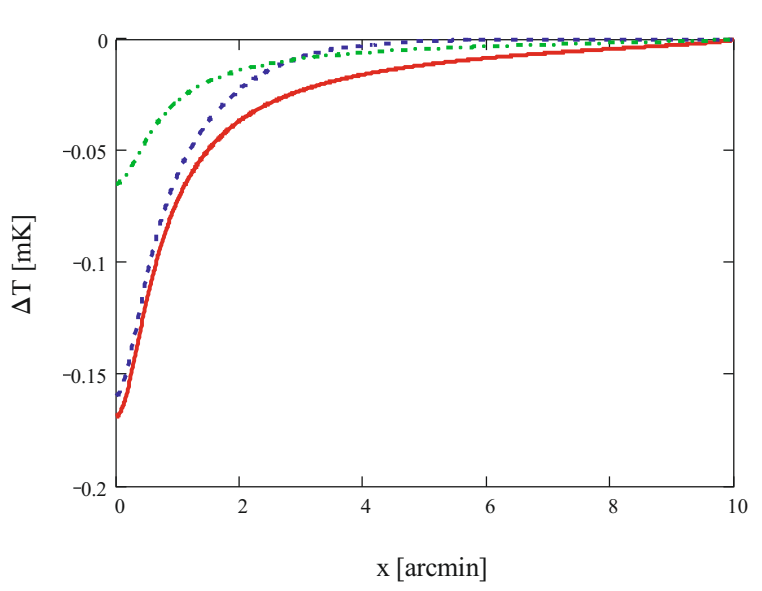

Fig. 3. SZ temperature decrements: for the isothermal beta model (solid line), for the non-isothermal beta model (dashed line), and for the isothermal beta model in which the value of the total SZ flux corresponds to the non-isothermal model (dot-dashed line).

Although the total SZ flux for the non-isothermal beta model is significantly lower than that for the isothermal model, attention should be drawn to a paper of Bielby \& Shanks (2007), where they truncated the isothermal beta model at the $2^{\prime}$ radius and still found a predicted SZ effect much larger than the level observed by WMAP, for a sample of clusters (Bonamente et al. 2006). Note that the approach of Afshordi et al. (2006) is different from that of Bielby \& Shanks (2007), in the former the X-ray data are mainly used to define a template to detect SZ decrements and then the SZ data and the X-ray temperature data alone are used to establish the gas densities.

\section{EUV emission from a baryonic shell}

Extreme ultraviolet (EUV) astronomy has produced substantial results in a wide variety of fields (e.g. Bowyer et al. 2000). The Extreme Ultraviolet Explorer (EUVE) has detected emission from a few clusters of galaxies in the 70-200 eV energy range.

Since baryonic shells around clusters are a possible location of missing cluster baryons and since the time scale of collisions between baryons and electrons is longer than the Hubble time in such shells, these cold electrons should be a source of extremeultraviolet emission.

In this section the contribution to the total cluster EUV emission from a baryonic shell is estimated for the Coma cluster. The Coma cluster is a rich, hot $\left(T_{\text {in }}=8 \mathrm{keV}\right)$, nearby $\left(D=100 h_{70}^{-1} \mathrm{Mpc}\right)$ galaxy cluster, which is often considered as a "standard cluster" due to the wealth of observational data available for this object.

The spectral surface brightness along a particular line of sight writes as

$b(E) \sim \int n_{\mathrm{e}}^{2}(\boldsymbol{r}) \Lambda\left(E, T_{\mathrm{e}}\right) \mathrm{d} l$

where $\Lambda\left(E, T_{\mathrm{e}}\right)$ is the spectral emissivity of the gas at observed energy $E$, and $T_{\mathrm{e}}$ is the electron temperature.

The spectral emissivity due to the electron-proton bremsstrahlung rate is given by

$\Lambda\left(E, T_{\mathrm{e}}\right) \sim \frac{1}{\sqrt{T_{\mathrm{e}}}} \exp \left(-\frac{E}{k_{\mathrm{B}} T_{\mathrm{e}}}\right)$.
For the sake of clarity, we propose that the hot gas is located inside the virial sphere. The electrons remain cold inside the baryonic shell, because the time scale of their collisions with the hot protons is longer than the Hubble time. The temperature of these electrons is almost the same as the preshock temperature, a plausible value is $T_{\text {ext }}=3 \times 10^{6} \mathrm{~K}$.

The gas density is given by the isothermal beta model (Eq. (1)) within the virial sphere, and the gas density inside the shell, which corresponds to missing baryons in the galaxy cluster (if $33 \%$ of baryons are missing), equals

$n_{\text {shell }}=0.5 \int_{0}^{R_{\mathrm{vir}}} n(r) 4 \pi r^{2} \mathrm{~d} r \cdot\left(\frac{4 \pi}{3} R_{\mathrm{s}}^{3}-\frac{4 \pi}{3} R_{\mathrm{vir}}^{3}\right)^{-1}$

where the center number density $n_{0}=3.4 \times 10^{-3} \mathrm{~cm}^{-3}$, the core radius $r_{\mathrm{c}}=290 \mathrm{kpc}, \beta=0.75$, the virial radius $R_{\mathrm{vir}}=2.8 \mathrm{Mpc}$ for a Coma-like cluster $\left(H_{0}=70 \mathrm{~km} \mathrm{~s}^{-1} \mathrm{Mpc}^{-1}\right.$ is adopted $)$.

The spectral surface brightness from the hot gas is

$b_{\mathrm{h}}(\theta) \sim \int_{-\Phi_{\max }(\theta)}^{\Phi_{\max }(\theta)} n_{0}^{2} \cdot\left(1+\frac{D^{2} \sin ^{2} \theta}{r_{\mathrm{c}}^{2} \cos ^{2} \Phi}\right)^{-3 \beta} \frac{\mathrm{e}^{-E / T_{\mathrm{in}}}}{\sqrt{T_{\mathrm{in}}}} \frac{D \sin \theta}{\cos ^{2} \Phi} \mathrm{d} \Phi$

where $\theta$ is the angle between the center of the cluster and the direction of interest, $\Phi_{\max }$ is defined by

$\Phi_{\max }=\operatorname{arctg}\left(\frac{\sqrt{R_{\mathrm{vir}}^{2}-D^{2} \sin ^{2} \theta}}{D \sin \theta}\right)$

where $D$ is the distance of the cluster.

The spectral surface brightness from the cold electrons is given by

if $\theta<R_{\text {vir }} / D$ :

$b_{\mathrm{c}}(\theta) \sim 2 \int_{\Phi_{\max }(\theta)}^{\Phi_{\max 2}(\theta)} n_{\text {shell }}^{2} \frac{\mathrm{e}^{-E / T_{\mathrm{ext}}}}{\sqrt{T_{\mathrm{ext}}}} \frac{D \sin \theta}{\cos ^{2} \Phi} \mathrm{d} \Phi$

if $\theta \geq R_{\text {vir }} / D$ :

$b_{\mathrm{c}}(\theta) \sim \int_{-\Phi_{\max 2}(\theta)}^{\Phi_{\max 2}(\theta)} n_{\text {shell }}^{2} \frac{\mathrm{e}^{-E / T_{\text {ext }}}}{\sqrt{T_{\text {ext }}}} \frac{D \sin \theta}{\cos ^{2} \Phi} \mathrm{d} \Phi$

where $\Phi_{\max 2}$ is

$\Phi_{\max 2}=\operatorname{arctg}\left(\frac{\sqrt{R_{\mathrm{s}}^{2}-D^{2} \sin ^{2} \theta}}{D \sin \theta}\right)$.

The normalized spectral surface brightness writes as

$B(\theta)=\frac{b_{\mathrm{h}}(\theta)+b_{\mathrm{c}}(\theta)}{b_{\mathrm{h}}(0)+b_{\mathrm{c}}(0)}$.

For the Coma cluster, the normalized spectral brightness at observed energy $0.1 \mathrm{keV}$ is plotted in Fig. 3, where $1^{\circ}$ is $1.7 \mathrm{Mpc}$. Figure 3 shows the presence of an extreme-ultraviolet emission halo around the galaxy cluster without the X-ray emission from it, because only the cold electrons are situated in the baryonic shell. 


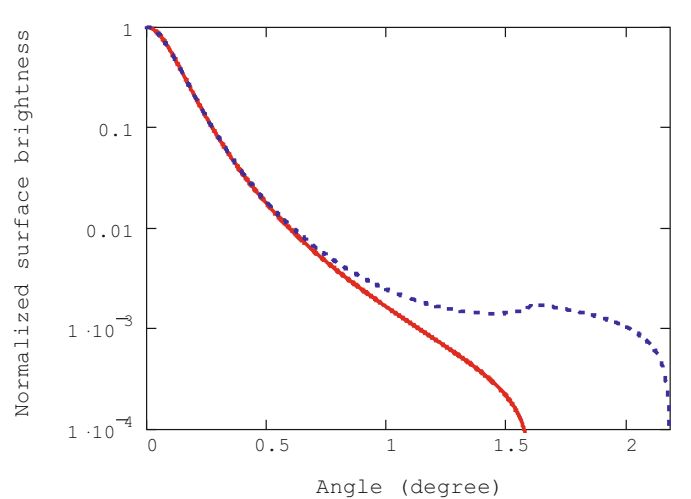

Fig. 4. Normalized surface brightness for the Coma cluster: the hot gas (solid line), the hot gas + the baryonic shell (dashed line).

\section{Discussion and conclusions}

Cluster gas mass fractions as inferred from X-ray observations have been used as a probe of the universal ratio of baryon to total mass densities $\Omega_{\mathrm{B}} / \Omega_{\mathrm{M}}$ (e.g. White et al. 1993; David et al. 1995; Evrard 1997; Roussel et al. 2000; Allen et al. 2002; Ettori 2003; Allen et al. 2004). But the result of recent measurements of $\mathrm{SZ}$ effect and X-ray studies is that a significant fraction $35 \% \pm 8 \%$ of the baryons is missing from the ICM (Ettori 2003; LaRoque et al. 2006; Vikhlinin et al. 2006; Afshordi et al. 2007; McCarthy et al. 2007). A possible explanation is that a significant fraction of baryonic mass may be hidden in a form that is difficult to detect with ordinary observational means.

The problem is unlikely to be associated with the conventional cluster mass estimates at least within the Abell radius, which rely upon the hydrostatic equilibrium hypothesis for the dynamical state of clusters in the computation of their total masses (e.g. Wu \& Xue 1999). This point has been justified by the excellent agreement among the X-ray, optical and weak lensing determined cluster masses on Abell radius scales (e.g. Allen 1998; Wu et al. 1998). However how accurate the conventional cluster mass estimates will be in the outskirts of clusters where hydrostatic equilibrium becomes questionable remains unclear.

We conclude within the framework of hydrostatic theory that the gas is considerably more extensively distributed than the mass in the cluster. Therefore a significant fraction of the ICM baryons may be located in shells around galaxy clusters.

In this paper the formation of baryonic shells around clusters is considered within the framework of the kinetic theory. Baryonic shells can be formed in this way for galaxy clusters with masses $\left(M>1.2 \times 10^{15} M_{\odot}\right)$.

We propose that the low baryon fraction inferred from the SZ effect observations in the CMB maps of WMAP data (Afshordi et al. 2007) can be explained by the deviation from thermal equilibrium in the cluster outskirts. The electrons and protons do not reach equipartition there. This warm phase (with electron temperature $T_{\mathrm{e}} \sim 0.1 \mathrm{keV}$ and with high proton temperature) of the ICM in the cluster outskirts does not contribute appreciably to the SZ signal. The necessary condition on the cluster mass for the concealment of the cluster missing baryons in a baryon shell is fulfilled because cluster masses are comparable to the value of the characteristic mass $M=e^{4} /\left(m_{\mathrm{p}}^{3} G^{2}\right)=$ $1.3 \times 10^{15} M_{\odot}$.
We considered a non-isothermal beta model for the ionized gas distribution of a cluster and showed that the total SZ flux of a cluster differs by a factor of 2.6 from that computed with a single-temperature beta model.

The cold electrons in shells around galaxy clusters should be a source of extreme-ultraviolet emission. Observations of cluster outskirts can shed light on a location of missing baryons.

Other possibilities that have been proposed to solve the cluster missing baryon problem are: diffuse intracluster light, which would be challenging to detect observationally, but could potentially hide significant amounts of stellar mass in the vast intracluster space (Lin \& Mohr 2004; Gonzales et al. 2005; Zibetti et al. 2005; Monaco et al. 2006); hiding baryons in the form of cold and compact dark baryonic clouds from local cooling instabilities within the ICM (Dwarakanath et al. 1994); evaporation of baryons out of the virial radius of the cluster (Loeb 2007); the correct value of $\Omega_{\mathrm{M}}$ is higher than the best-fit WMAP value (McCarthy et al. 2007).

Acknowledgements. I am grateful to Florence Durret and Roya Colin Mohayaee for valuable discussions and to the referee for constructive comments.

\section{References}

Afshordi, N., Lin, Y.-T., Nagai, D., et al. 2007, MNRAS, 378, 293 Allen, S. W. 1998, MNRAS, 296, 392

Allen, S. W., Schmidt, R. W., \& Fabian, A. C. 2002, MNRAS, 334, L11 Allen, S. W., Schmidt, R. W., Ebeling, H., et al. 2004, MNRAS, 353, 457 Bertschinger, E. 1985, ApJS, 58, 39

Bielby, R. M., \& Shanks, T. 2007, 382, 1196

Bonamente, M., Joy, M. K., LaRoque, S. J., et al. 2006, ApJ, 647, 25

Bowyer, S., Drake, J. J., \& Vennes, S. 2000, ARA\&A, 38, 231

Cavaliere, A., \& Fusco-Femiano, R. 1976, A\&A, 49, 137

Crain, R. A., Eke, V. R., Frenk, C. S., et al. 2007, MNRAS, 377, 41

David, L. P., Jones, C., \& Forman, W. 1995, ApJ, 445, 578

Dunkley, J., Komatsu, E., Nolta, M. R., et al. 2008 [arXiv:0803.0586]

Dwarakanath, K. S., van Gorkom, J. H., \& Owen, F. N. 1994, ApJ, 432, 469

Eke, V. R., Cole, S., \& Frenk, C. S. 1996, MNRAS, 286, 263

Eke, V. R., Navarro, J. F., \& Frenk, C. S. 1998, ApJ, 503, 569

Ettori, S. 2003, MNRAS, 344, L13

Evrard, A. E. 1997, MNRAS, 292, 289

Frenk, C. S., White, S. D. M., Bode, P., et al. 1999, ApJ, 525, 554

Fox, D. C., \& Loeb, A. 1997, ApJ, 491, 459

Gonzales, A. H., Zabludoff, A. I., \& Zaritsky, D. 2005, ApJ, 618, 195

Jones, C., \& Forman, W. 1984, ApJ, 276, 38

Kay, S. T., Thomas, P. A., Jenkins, A., et al. 2004, MNRAS, 355, 1091

Kravtsov, A. V., Nagai, D., \& Vikhlinin, A. 2005, ApJ, 625, 588

LaRoque, S. J., Bonamente, M., Carlstrom, J. E., et al. 2006, ApJ, 652, 917

Lieu, R., Mittaz, J. P. D., \& Zhang, S.-N. 2006, ApJ, 648, 176

Lin, Y.-T., \& Mohr, J. J. 2004, ApJ, 617, 879

Loeb, A. 2007, J. Cosmology Astropart. Phys., 3, 1

McCarthy, I. G., Bower, R. G., \& Balogh, M. L. 2007, MNRAS, 377, 1457

Meszaros, P., \& Ostriker, J. P. 1983, ApJ, 273, L59

Monaco, P., Murante, G., Borgani, S., et al. 2006, ApJ, 652, L89

Navarro, J. F., Frenk, C. S., \& White, S. D. M. 1996, ApJ, 462, 563

Refregier, A., Spergel, D. N., \& Herbig, T. 2000, ApJ, 531, 31

Roussel, H., Sadat, R., \& Blanchard, A. 2000, A\&A, 361, 429

Sarazin, C. L. 1986, Rev. Mod. Phys., 58, 1

Spitzer, L. 1956, Physics of Fully Ionized Gases (New York: Interscience)

Sunyaev, R. A., \& Zeldovich, Ya. B. 1980, ARA\&A, 18, 537

Takizawa, M., \& Mineshige, S. 1998, ApJ, 499, 82

Vikhlinin, A., Kravtsov, A., Forman, W., et al. 2006, ApJ, 640, 691

White, S. D. M., Navarro, J. F., Evrard, A. E., et al. 1993, Nature, 366, 429

Wu, X.-P., \& Xue, Y.-J. 2000, MNRAS, 311, 825

Wu, X.-P., Chiueh, T., Fang, L.-Z., et al. 1998, MNRAS, 301, 861

Zhang, Y.-Y., Bohringer, H., Finoguenov, A., et al. 2006, A\&A, 456, 55

Zibetti, S., White, S. D. M., Schneider, D. P., et al. 2005, MNRAS, 358, 949 